\title{
Study on Model-Based System Engineering in Satellite Layout
}

\author{
Jiangyong Wang ${ }^{\mathrm{a}}$, Fa Jing, Yongquan Xia and Chunsheng Xu \\ Institute of Telecommunication Satellite, China Academy of Space Technology, Beijing 100094, China
}

\begin{abstract}
For satellite layout, it is necessary to collate manually in order to obtain accurate product technical requirements information. It is difficult to track the technical status information. It is proposed that the application of model-based system engineering (MBSE) in the cooperative development of multi-disciplinary and multisectoral satellite layout was an effective way to solve these problems. Based on the four typical processes of layout design, this paper studied the requirements to occupancy design, electromechanical joint design, integration of Attitude and Orbit Control System (AOCS) equipment layout, definition and display of equipment expansion, and give the verification design of star sensor layout. The results show that this method is beneficial to the collaboration of all departments, and improve the design ability.
\end{abstract}

\section{Introduction}

With the increasing demand for satellites, satellite systems are becoming more complex and need higher standards, the index has been greatly improved. Many new technologies are facing challenges. They also have the characteristics of shortening development cycle and possibly changing the technological status in the process of development, which require that the impact of the changes be given quickly. In the traditional satellite development work, a large amount of information about product technical requirements is hidden in drawings and various documents. It must be sorted out manually to obtain accurate information of product technical requirements. It is difficult to track the technical status information. In the process of satellite development, it is necessary to collect all kinds of inputs, which are controlled by human, and the workload is huge. Then, the design work is carried out according to the sequence of factors been influenced.

Demand parameters rely on manual tracking step by step. As the input for follow-up research work, Demand parameters lack traceability in the process of definition, decomposition, allocation and verification, It also lack effective digital analysis and simulation method. They often start detailed design before the scheme is mature and stable, resulting in a large number of design rework and delays. In addition, there is no complete end-to-end requirement traceability and influence analysis, resulting in many design iterations and rework. Requirements/design/validation results are mainly recorded and transmitted in the form of documents, which makes it difficult to trace and trace the whole development process, and to assess the impact of changes timely and effectively when changes occur.
In the process of mechanical and electrical joint design, although some collaborative design work has been carried out, the situation of "separating wall" between different departments/specialties is still very prominent, lack of integrated collaborative development method and carriers, a large number of format conversion when exchanging data, and duplication of work lead to poor communication and transmission of information.

Lack of early model-based virtual verification, there is no closed-loop design, mainly through the indicators of parameters to verify the validation of the benchmark (generally based on empirical data). For example, in satellite development, according to the product configuration of Attitude and Orbit Control System(AOCS) specialty, the installation requirements of sensors and actuators for satellite AOCS specialty are given, and the whole satellite layout design is carried out to meet the requirements of AOCS specialty.

Three-dimensional model has been widely used in manufacturing and production, but its role in analysis is insufficient. It is necessary to expand the application of three-dimensional model, use three-dimensional model to carry out virtual simulation, and give information on the model for other subsystems to use. Taking the threedimensional digital satellite as the object, based on the traditional theory and method of physical sample satellite testing and analysis, the theoretical model of satellite pure digital simulation and comprehensive analysis is constructed. The reuse rate of the model is still very low, so it is necessary to reuse the model well and make the best use of the model, and provide a variety of data interfaces for other subsystems to call.

Model-based systems engineering has been applied in many fields. The National Aeronautics and Space Administration (NASA), the United States Department of

\footnotetext{
${ }^{\text {a }}$ Corresponding author: wangjy501@163.com
} 
Defense and the European Space Agency are actively applying the MBSE method, and have achieved results $[1$, ${ }^{2]}$. NASA has made great breakthroughs in the design of CubeSat $^{[3][4]}$ and FireSat ${ }^{[5]}$ using MBSE method, which also reflects the advantages of MBSE method in satellite development.

NASA JPL gave a speech entitled "OpenCAE Case Study: Europa Land Concept" on INCOSE International Workshop in 2019. Based on the Europa Lander project, this paper introduced the current model-based product development environment of NASA JPL ${ }^{[6]}$. MBSE has become a research and application hotspot in the field of system engineering in recent years ${ }^{[7]}$.

MBSE emphasizes the consideration of requirements, design and validation in the process of work. By establishing the relationship between validation model and requirement model, logical model, and other relevant models used in the design process, the validation coverage analysis is carried out to ensure that all projects meet the validation requirements. This paper studies the implementation of model-based engineering in satellite layout from four aspects, and takes star sensor integrated layout as an example to verify.

\section{Satellite Layout and Model-based Systems Engineering}

\subsection{Relevant Contents of Satellite Layout}

In satellite development, satellite configuration layout design is an important overall design work. The basic task of spacecraft configuration design is to combine satellite subsystems and their instruments and equipment into an internal and external space dimension coordination, to ensure the realization of spacecraft functions, and to meet the installation requirements and capabilities of instruments and equipment in each subsystem. The mechanical environment of launch vehicle is favorable to the development of spacecraft and the growth of payload capability of spacecraft. Satellite configuration is not only the main force-bearing component for designing satellite structure subsystems, but also the overall shape, volume and size allocation, overall layout and mass characteristics calculation of instruments and equipment of satellite subsystems ${ }^{[8]}$.

The overall layout is an important work in satellite configuration $^{[9]}$. Arrange a reasonable layout to meet the various needs of satellite missions. Layout includes: instrument and equipment layout design (including AOCS sensors); propulsion system (pipeline) layout design; cable network routing design; layout design outside the satellite; and layout analysis (sensor field of view analysis, interference analysis, mass characteristics calculation), and so on.

\subsection{Concepts and Processes of Model-based System Engineering}

INCOSE, which first proposed the concept of MBSE, gave the following definition: "Model-based system engineering is a formal modeling tool that can support activities such as requirements, design, analysis, validation and confirmation from the conceptual design stage, and continue throughout the whole development process and the subsequent life cycle stage" [10]. MBSE method takes model as the basis of system description and realizes the whole process from conceptual design, scheme design, experimental verification to engineering implementation with visualized model. Compared with document-centred system engineering method, MBSE has the advantages of high understanding and communication efficiency, easy data acquisition, good traceability of technical status, and integration of design and verification ${ }^{[11][12]}$.

In general, MBSE can be cut in practical applications according to the overall process of requirements, functions, logic and physics.

1) Requirements: Describe the requirements that should be met from the perspective of system black box. Requirement model refers to the set of requirements from top to the bottom of the system, and the logical relationship between them.

2) Function: The technical function of the system proposed in response to the demand is the white box of the demand. The functional framework describes all the functions of the system and their interfacing relationships. 3) Logic: Describe the relationship of system composition, and the interfacing relationship among system, subsystem and component.

4) Physics: The Final Three-Dimensional Definition of Products.

\section{Application of Model-based System Engineering in Layout Design}

\subsection{Requirements trace back to space occupancy design}

The traditional document-based requirement management mode is adopted in aerospace model development. The requirement mode has many limitations. It is difficult to change, maintain and track the change of users' requirement and its impact on the system in time, and the requirement comes from different documents. And the development of requirement management technology has solved this problem. Requirement management generally includes requirement definition, requirement allocation, requirement validation and traceability management. Traceability-oriented requirement management and control can drive and correlate requirements to downstream engineering activities.

In the design of satellite configuration layout, different requirements are defined and allocated: boundary constraints of large-scale systems (such as carry rocket), design prohibition zone, AOCS constraints, interface constraints, fixture tooling constraints, etc., and then subdivided to verify the requirements of subdivision, figure1. 


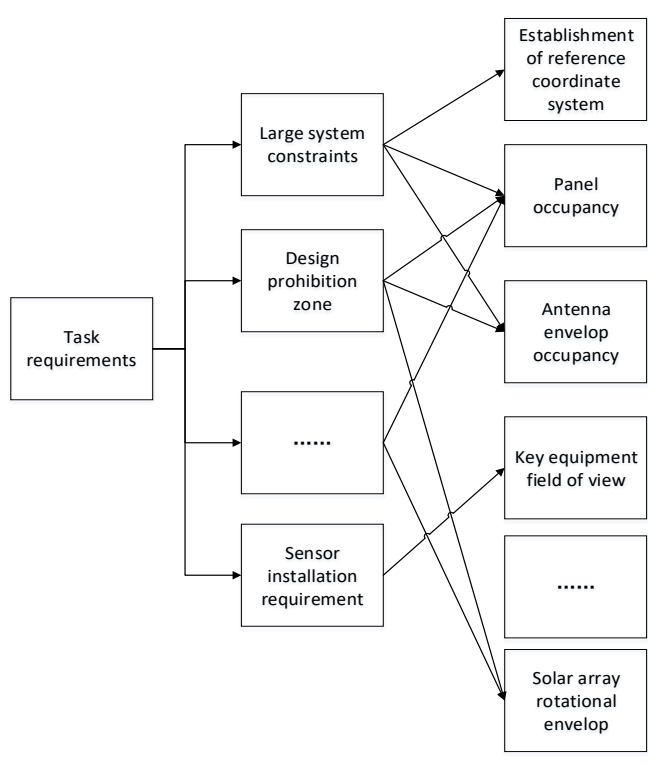

Figure 1. Construction of requirements model

According to the occupancy design, the requirements are preliminarily verified. The space occupancy of the whole satellite is determined by the whole satellite coordinate system, the structure coordinate system and the local coordinate system. The space static envelope is used to determine the space occupancy of the large parts, and the motion envelope is used to determine the moving area of the moving parts. For the key single equipment, the field of view surface is established with the constraint of the viewpoint and the main axis of the field of view. Establish the traceability pedigree of demand and occupancy design, and realize the traceability control of requirement.

\subsection{Mechatronics Joint Design Based on Electrical Principle}

Multi-specialty joint simulation of electrical and mechanical engineering is carried out in cable design. According to the requirements of satellite overall design, we customize and manage single or multi-level classification of electrical parts, define standard electrical connection attributes, form a unified Library of electrical components, and ensure the unification of data of electrical components in the whole professional design. Electrical schematic diagram is established to cover the whole process of system principle design. Attributes and technical parameters are automatically captured in the schematic diagram. Connectivity and quality of the schematic diagram are analyzed. Synchronization with $3 \mathrm{D}$ design is achieved through business rules. Improve the quality of electrical system and data continuity, make the electrical 2D-3D design process realize the integration based on database, design and optimize the accuracy of electrical 3D harness channel/cable channel in the overall product context. Through intuitive threedimensional geometric wiring harness routing design, the structure layout of wiring harness (wiring harness structure catalogue, trunk routing, branch point setting, branch routing, etc.) is determined. By laying electrical signal wiring harness, the transmission path of electrical signal is established, and the function of electrical logic design of contact list is realized. Geometric wire harness and electrical wire harness are combined to form the whole wire harness design system, which provides information about the mass, center of mass and moment of inertia of satellite wire harness system. Through the three-dimensional annotation of electrical wiring harness, it can guide the production and assembly of follow-up workshop, and realize the design of full threedimensional electrical system; or form flattening drawings, which are compatible with the status that some workshops need two-dimensional drawings to guide production. The real-time synchronization between the harness digital mockup and the flattening digital mockup is realized.

In view of the trend of three-dimensional geometric wire harness, the calculation rules of the bending radius of the wire harness, the design points of cable slack and the design elements of cable color are encapsulated and integrated. Table 1 is an example table of design elements, which becomes the corresponding design guide template, and is integrated with the mechanical three-dimensional design software to support mechanical design. Based on this, the staff can input the cable network design data quickly, reduce the workload of manual calculation and improve the overall efficiency of three-dimensional cable design.

Table 1. Digital cable design elements

\begin{tabular}{|l|c|l|l|}
\hline \multicolumn{1}{c|}{$\begin{array}{c}\text { Design } \\
\text { activities }\end{array}$} & $\begin{array}{c}\text { Elements } \\
\text { number }\end{array}$ & $\begin{array}{c}\text { design } \\
\text { elements }\end{array}$ & $\begin{array}{c}\text { DesignEss } \\
\text { entials }\end{array}$ \\
\hline \multirow{4}{*}{$\begin{array}{l}\text { Harness } \\
\text { control }\end{array}$} & 1 & $\begin{array}{l}\text { wire } \\
\text { diameter }\end{array}$ & $\bullet \ldots$ \\
\cline { 2 - 4 } & 2 & $\begin{array}{l}\text { Bend } \\
\text { radius }\end{array}$ & $\bullet \ldots$ \\
\cline { 2 - 4 } & 3 & slack & $\bullet \ldots$ \\
\cline { 2 - 4 } & 4 & $\ldots$ & $\bullet \ldots$ \\
\hline
\end{tabular}

\subsection{Integrated Layout Design from Control Parameters to Attitude Control Equipment}

The satellite AOCS consists of attitude sensor, controller and actuator. Sensors measure satellite attitude angle and attitude angular velocity. Controller calculates control force and control moment according to the information output by sensors, and produces corresponding control effect by actuator. The layout of control system equipment is a multidisciplinary and complex issue involving control, machinery, optics and so on. Each device of the control system has a design input to the layout, including the requirements of cable distance between devices, field of view of sensors, pointing requirement of actuator (thruster), layout space of controller cabin, and precision measurement requirement of sensors, figure 2 . 


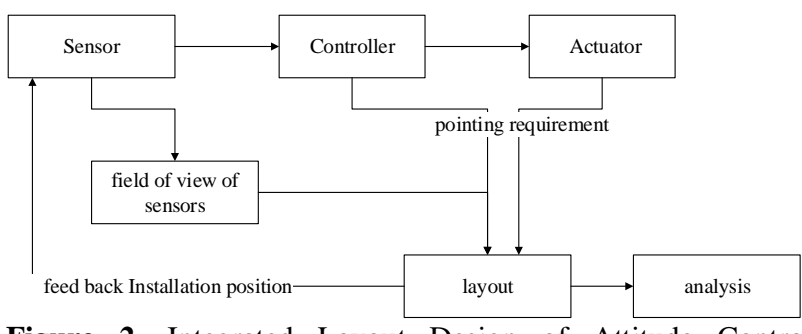

Figure 2. Integrated Layout Design of Attitude Control Equipment

Attitude control sensors and thrusters in satellites require no other parts to be shielded in their field of view, so they can be arranged accordingly. According to the occlusion results of each sensor, the effectiveness of the combined sensor to the target is obtained. Sensor field of view analysis includes field of view occlusion analysis and sensor effectiveness analysis. Field-of-view occlusion analysis can be simplified to determine whether the cone at a point in a certain direction interferes with other satellite components or other targets. A single sensor is equivalent to a point light source. Starting from the light source, the visible star surface elements are projected onto the designated polar coordinate plane to analyze the visible region of the field of view at all angles, and then calculate the occlusion of the sensor at a certain time. The field of view can be represented by a cone.

For actuators such as thrusters, the space coordinates and azimuth angles of the thruster's thrust points are placed in the layout, and the thrust and moment are calculated to determine the layout after meeting the requirements. After the layout is determined, the position and mass characteristics of the equipment layout are fed back to the control for further analysis.

\subsection{Extended Definition and Integrated Display of Equipment}

Satellite layout involves a large number of instruments and equipments, which are defined more extensively. Combining form data with attribute definition in threedimensional model. The mechanical interface (including basic information, installation information, MCI information, etc.), thermal interface (basic thermal performance, temperature measurement point, heat consumption, temperature range) and remote control information include basic information of remote measurement parameters (parameter code, parameter name, parameter category). The basic information of remote control command (instruction code, instruction name, instruction type, etc.), parameter processing method, instruction criterion and channel information are all managed in a unified way. On the basis of equipment model used in layout design, the application scope of three-dimensional model is extended.

Using the model to carry out simulation analysis, we try our best to solve the problem of product assembly at the design stage, eliminate the hidden danger of downstream product manufacturing, and avoid large rework. In addition to the need for assembly design at the design stage, we also need to evaluate the assemblability of the product at the later stage of product design, as well as the simulation of the production line and launching site.
Practical simulation, human-machine efficiency analysis, improve the ability of satellite disassembly simulation and human-machine simulation.

The virtual simulation is carried out by using threedimensional model, and the information given on the model is used by other professionals to realize the automatic reading of the definition information of instrument and equipment model. Taking the threedimensional digital satellite as the object, based on the traditional physical sample satellite test results and analysis theory, the satellite digital simulation and comprehensive analysis correction model is constructed, including three-dimensional structure analysis model, thermal performance analysis model, etc.

In order to meet the requirement of other specialties to design by using three-dimensional model, the threedimensional model is automatically transformed into a lightweight integrated display model. The information specialty uses it to design the sequential arrangement of matrix cables and bus cables. The electrical specialty uses it to carry out voltage drop analysis, power supply and distribution simulation analysis, and the test specialty can view the distribution location, superior designers can review the layout of equipment.

\section{Star Sensor Layout Verification}

Verification work is carried out to control the layout design of star sensors on satellites. Star sensor is a kind of sensitive device. It is a precise instrument to measure three-axis attitude of spacecraft by stellar vector. It gives three-axis attitude parameters of coordinate system relative to inertial system in quaternion form, and completes the measurement of satellite attitude angle. According to the parameter requirements of satellite star sensor, the requirement parameters such as field of view position are sorted out, and the requirement definition and control are carried out. The space layout of the system is defined by mapping the three-dimensional model of star sensor's position requirement, and the star sensor is defined as the installation point and the field of view, and the field of view requirement is defined from the field of view,figure 3 . The results were input to the star-sensitive black-box model, and the black-box model was used to calculate and output various kinds of information.

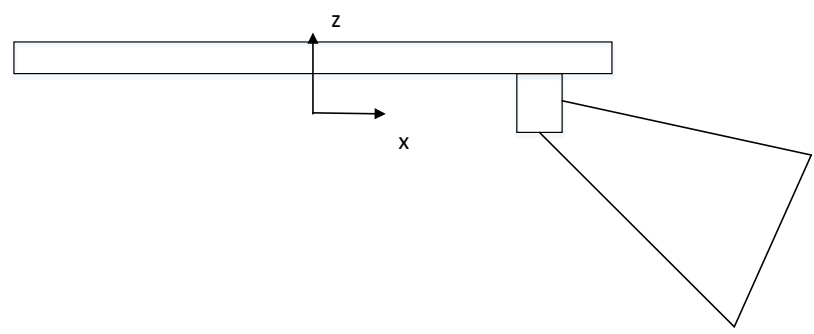

Figure 3. Star sensor Spatial Layout 


\section{Conclusion}

Model-based system engineering has developed from exploratory research to application abroad, and there are also some research results in $\mathrm{China}^{[13]}$. This paper studies the application of model-based system engineering in satellite layout from four aspects, and validates it with the example of star sensor layout integration. It is proved that this method is of great significance to the improvement of satellite overall design level.

\section{References}

1. Kevin Vipavetz,Douglas Murphy,Samantha Infeld Model-based systems engineering pilot program at NASA Langley//AIAA SPACE 2012 Conference\&Exposition.Washington D.C.:AIAA,(2012)

2. Nadia A Tepper.Exploring the use of Model-Based Systems Engineering(MBSE)to develop systems architecture in naval ship design.Cambridge:Massachusetts Institute of Technology,(2010)

3. Spangelo S C,Cutler J,Anderson L,et a1.Model based systems engineering(MBSE)applied to Radio Aurora Explorer(RAX)CubeSat mission operational scenarios//Aerospace conference.New York:IEEE,(2013)

4. Kaslow,D.Anderson,L.Asundi, S, et a1.Developing a CubeSat Model-Based System Engineering (MBSE)
Reference Model - interim status. Montana:IEEE Aerospace Conference,(2015)

5. Larson w J.Applied space systems engineering.New York:McGrew - Hill Learning Solution,(2009)

6. Eric W Brower.OpenCAE Case Study:Europa Land Concept//2019 Annual INCOSE International Workshop, Torrance,CA,USA,(2019)

7. Wang Kunsheng, Yuan Jianhua,Chen Hongtao,et a1.Investigation and practice of foreign systematic engineering method based on model.Aerospace China, 11(2012)

8. Peng chengrong.Overall Design of Spacecraft.Beijing:Science and technology of China press,(2011)

9. $\mathrm{Xu}$ fuxiang,Lin huabao,Hou shenyuan.Introduction to Satellite Engineering.Beijing:China Astronautic Publishing House, (2003)

10. HAN Fengyu,LIN Yiming,FAN Haitao.Research and Practice of Model-Based Systems Engineering in Spacecraft Development.Spacecraft Engineering, 3(2014)

11. ZHANG Youshan,YANG Lei,WANG Ping,et a1.Discussion 0n Application of Model-based Systems

12. Engineering Method to Human Spaceflight Mission. Spacecraft Engineering, 10(2014)

13. FeiFei Chen,Gang Zhao,Yong Yu,et a1. Modelicabased modeling and simulation of satellite On-orbit deployment and attitude control. Proceedings of the 2016 4th International Conference on Machinery, Materials and Information Technology Applications,(2017) 